\title{
Variações de gradação na reinstanciação da atitude em textos ficcionais traduzidos
}

\section{$V$ ariations of gradation in the re-instantiation of attitude in translated fictional texts}

\author{
Cliver Gonçalves Dias* \\ Universidade Federal de Minas Gerais \\ Belo Horizonte, Minas Gerais, Brasil
}

Resumo: O objetivo deste estudo foi investigar variações na gradação dos valores atitudinais (MARTIN; WHITE, 2005) em duas narrativas traduzidas. Adotou-se a perspectiva semântico-discursiva da estrutura genérica dos textos (MARTIN, 1992; MARTIN; ROSE, 2003, 2008) juntamente com a perspectiva de tradução como reinstanciação do texto-fonte (SOUZA, 2010). As narrativas selecionadas para este estudo foram Things Fall Apart (1994[1958]) e Arrow of God (1989[1964]), ambas escritas por Chinua Achebe. Suas reinstanciações em português brasileiro são O mundo se despedaça (2009[1983]) e A flecha de Deus (2011), pela tradutora Vera Queiroz da Costa e Silva. De cada narrativa, foram selecionados três conjuntos de fases discursivas de diferentes estágios discursivos. Para cada conjunto de fases do texto-fonte, selecionou-se o conjunto de fases equivalente nos textos traduzidos. Os procedimentos de análise foram divididos em duas etapas. $\mathrm{Na}$ primeira, os recursos que realizam gradação nos textos-fonte e textos traduzidos foram identificados e classificados de acordo com seu tipo e grau de intensificação ou prototipicidade. Na segunda etapa, as análises dos textos-fonte e traduzidos foram contrastadas para identificação de variações no acoplamento e/ou na calibragem da gradação dos valores atitudinais reinstanciados. Os resultados mostram que os textos traduzidos são menos graduados do que os textos-fonte, principalmente em relação aos valores de julgamento. Essa diminuição ocorre tanto pelo não acoplamento de recursos de gradação na reinstanciação de determinados valores quanto pelo menor grau de calibragem da gradação nas escolhas lexicais.

Palavras-chave: Variações semânticas. Valores atitudinais. Gradação. Reinstanciação. Narrativas traduzidas.

\begin{abstract}
This study aimed to investigate variations in the gradation of attitudinal values (MARTIN; WHITE, 2005) in two translated narratives. It adopted the discourse semantics perspective of the generic structure of texts (MARTIN, 1992; MARTIN; ROSE, 2003, 2008) and the perspective of translation as the reinstantiation of the source-text (SOUZA, 2010). The selected narratives are Things Fall Apart (1994[1958]) and Arrow of God (1989 [1964]), both written by Chinua Achebe. Their re-instantiations in Brazilian portuguese are O mundo se despedaça (2009[1983]) and A flecha de Deus (2011), both translated by Vera Queiroz da Costa e Silva. Three groups of discursive phases were selected from different discursive stages of each narrative. For each group of phases from the source-text, the equivalent group of phases in the translated texts was selected. The analysis was divided in two steps. In the first step, the resources realizing gradation in both source and translated texts were identified and classified according to the type and degree of intensification or prototypicality. In the second step, the analyses of the source and translated texts were contrasted for identification of variations in the coupling and/or commitment of gradation of the attitudinal values reinstantiated. Results show that the translated texts are less intensificated than their source-texts, especially regarding judgement values. This reduction stems from both the non-coupling of gradation resources in the re-instantiation of some values and the lower degree of gradation commitment of lexical choices.
\end{abstract}

Keywords: Semantic variations. Attitudinal values. Gradation. Re-instantiation. Translated narratives.

*Doutorando do Programa de Pós-Graduação em Estudos Linguísticos da Universidade Federal de Minas Gerais. E-mail: cliver.dias@gmail.com. 


\section{INTRODUÇÃO}

Recentes pesquisas dos Estudos da Tradução (CRISTÓFARO, 2018; CHANG, 2017) têm investigado a reinstanciação da VALORAÇÃO ${ }^{1}$ (MARTIN; WHITE, 2005) em narrativas traduzidas com o objetivo de explorar variações semânticas no acoplamento e/ou na calibragem dos significados reinstanciados nos textos traduzidos. Trata-se de uma perspectiva de tradução interlinguística desenvolvida por Souza (2010) com base no modelo semântico-discursivo de língua de Martin (1992, 2008).

Embora de diferentes perspectivas, Cristófaro (2018), Dias e Magalhães (2017), Blauth (2015), Munday (2012) e Rosa (2008) mostram que o sistema da GRADAÇÃo é produtivo para a investigação de variações na tradução de textos ficcionais. Alinhado à perspectiva de Cristófaro (2018), o objetivo deste estudo foi investigar variações de gradação em duas narrativas traduzidas da língua inglesa para o português. Indagou-se: Quais tipos de variação de gradação ocorreram nas duas narrativas? Quais valores atitudinais tiveram seu grau de intensificação ou prototipicidade ampliado ou reduzido? Quais implicações essas variações tiveram para a reconstrução das narrativas?

A metodologia desenvolvida para este estudo está fundamentada não somente nas orientações de Martin e White (2005), que servem de parâmetro geral para o estudo da VALORAÇÃO em qualquer gênero, mas também nas orientações de Macken-Horarik (2003) e Macken-Horarik e Isaac (2014), que se concentram especificamente no estudo da VALORAÇÃO em narrativas. No campo disciplinar dos Estudos da Tradução, adota-se o modelo de análise contrastiva de Souza (2010), desenvolvido com base no mesmo modelo de língua do qual a VALORAÇão é parte.

Juntamente com esta Introdução, este artigo está composto por cinco seções. $\mathrm{Na}$ seção 2, são apresentados os conceitos básicos da VALORAÇão e de seu modelo de língua, bem como são resenhados os resultados de trabalhos empíricos dos Estudos da Tradução sobre o tema aqui investigado. A terceira seção está dedicada aos procedimentos de seleção, delimitação e análise das narrativas. Os resultados e sua discussão são apresentados na seção 4. Por fim, na seção 5, são feitas considerações acerca do estudo realizado e sugestões para trabalhos futuros.

\section{REFERENCIAL TEÓRICO}

O modelo de língua adotado neste estudo foi proposto por Martin (1992) e é reapresentado em Martin e Rose (2003, 2008). Trata-se de um modelo que se fundamenta na Linguística Sistêmico-Funcional (HALLIDAY, 1994; HALLIDAY; MATTHIESSEN, 2004) para modelar a construção dos significados acima da oração. Segundo esse modelo, os significados são construídos pela coadunação de três metafunções (ideacional, interpessoal e textual) em diferentes estratos de abstração

\footnotetext{
1 O nome da rede de sistemas é sempre grafado em tipologia versalete. Os sistemas são grafados em versalete sempre que referidos como sistema.
} 


\section{das Letras}

(fonologia/grafologia, léxico-gramática, semântica discursiva e gênero). Ilustra-se a organização do modelo de Martin e Rose (2003) na Figura 1.

Figura 1: Modelo de língua

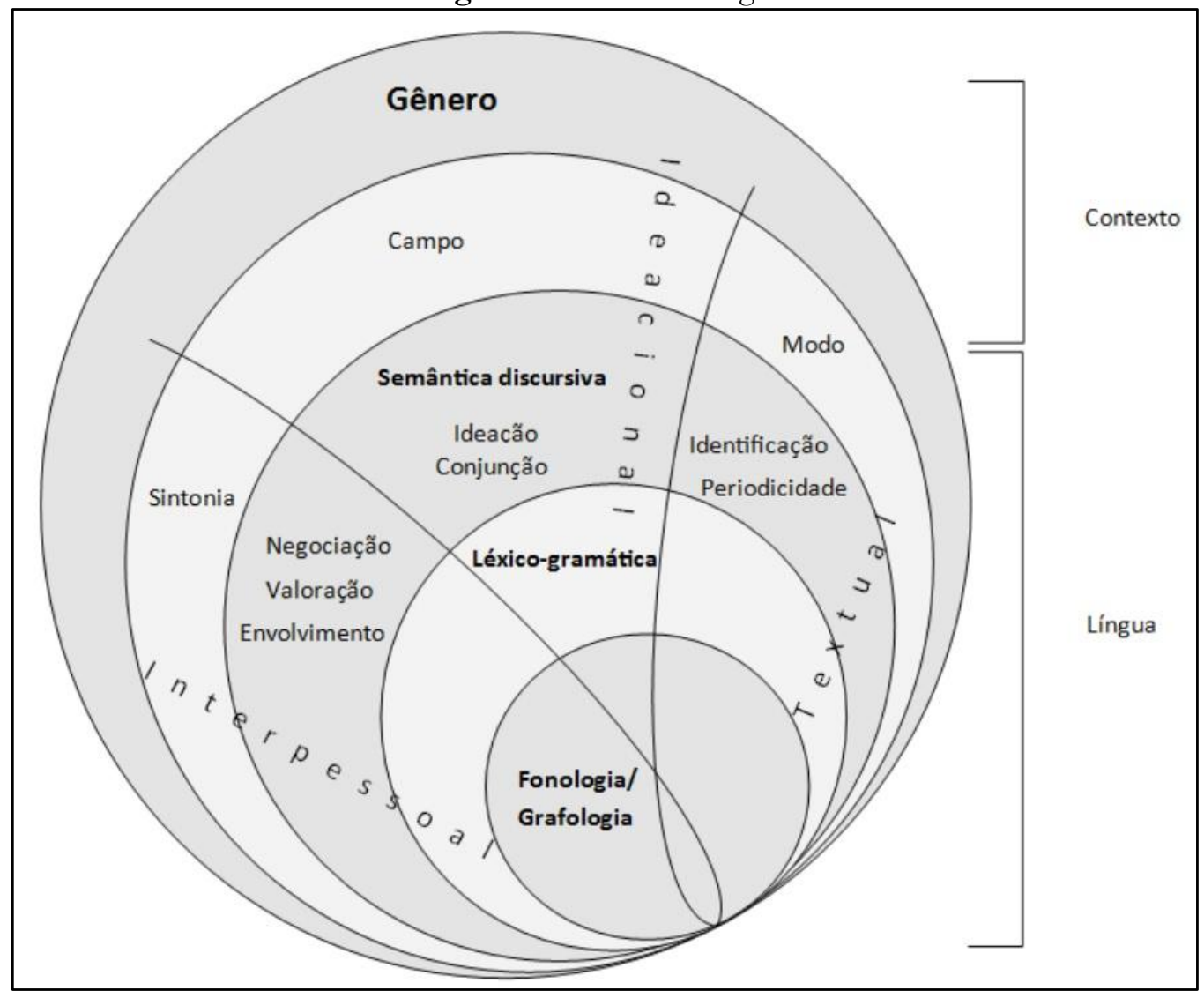

Fonte: Adaptado de Martin e White (2005).

Com base nesse modelo de língua e principalmente na estrutura genérica dos textos, Rothery e Stenglin (1997) definem os estágios discursivos do gênero narrativa como 1) uma "mini-explicação" que fornece ao leitor as informações básicas necessárias à compreensão do problema, tecnicamente denominada Orientação $\left.{ }^{2}, 2\right)$ uma "ruptura" da usualidade dos eventos na forma de um problema a ser resolvido, a Complicação, e 3) o (re)estabelecimento da usualidade dos eventos após o problema, a Resolução. Rothery e Stenglin (1997) apresentam mais um estágio (Avaliação), no entanto, o autor deste se afilia à perspectiva de Macken-Horarik (2003) ao considerar que a avaliação perpassa todos os estágios discursivos da narrativa.

Além da concepção de estágios discursivos, é também relevante a concepção de fase discursiva. De acordo com Martin e Rose (2008), as fases discursivas são um padrão

2 Assim como em Rothery e Stenglin (1997) e Macken-Horarik (2003), adota-se o uso de inicial maiúscula para a identificação técnica dos estágios discursivos. 
de configurações linguísticas que permitem considerar determinada porção de texto como "subestágios", unidades semântico-discursivas dentro dos estágios. Martin e Rose (2008) sublinham que, diferentemente dos estágios discursivos, as fases discursivas são muito diversificadas e, geralmente, próprias de cada texto.

Conforme apresentado em Martin e White (2005), a VALORAÇÃo é um conjunto de sistemas da metafunção interpessoal no estrato semântico-discursivo da linguagem verbal. Esses sistemas permitem identificar os recursos linguísticos usados pelos falantes/escritores para construir valores e crenças em seus textos, para posicionar-se e posicionar seus leitores prospectivos em relação a esses valores e crenças, bem como para ampliar ou diminuir o grau tanto dos valores e crenças quanto dos posicionamentos. Os recursos que constroem os valores e crenças são agrupados no sistema da ATITUDE, os recursos de posicionamento no sistema do COMPROMETIMENTO e os de ampliação ou redução no sistema da GRADAÇÃO. Neste artigo, é dado maior enfoque aos recursos de gradação, especificamente em relação aos valores atitudinais.

O sistema da GRADAÇÃO reúne dois tipos de recursos linguísticos de ampliação e redução do grau dos valores e crenças, os do tipo força e os do tipo foco. A gradação de força incide sobre elementos graduáveis quanto a sua intensidade, massa, extensão e volume. Um exemplo de gradação de força é "muito inteligente", no qual o intensificador "muito" eleva o valor de "inteligente". Já a gradação de foco incide sobre elementos graduáveis quanto a sua prototipicidade. Um exemplo é "um vestido de seda legítima", no qual o epíteto "legítima" coloca o valor "seda" no centro de prototipicidade de sua categoria.

Um traço peculiar da gradação é que ela pode ser realizada tanto de forma isolada (por um morfema, palavra ou grupo de palavras) quanto de forma fusionada (pela escolha lexical). Os exemplos apresentados no parágrafo antecedente são de recursos isolados, as palavras "muito" e "legítima", graduando determinados valores (inteligente, seda). Exemplos de gradação fusionada são as escolhas lexicais "gênio" e "lindo", que, de acordo com determinada comunidade de valores compartilhados, estão em um grau mais elevado em relação às escolhas "inteligente" e "bonito", respectivamente. Além disso, os valores atitudinais podem ser intensificados por meio de repetição. A Figura 2 ilustra a organização do sistema da GRADAÇÃO.

Figura 2: Sistema da GRADAÇÃO

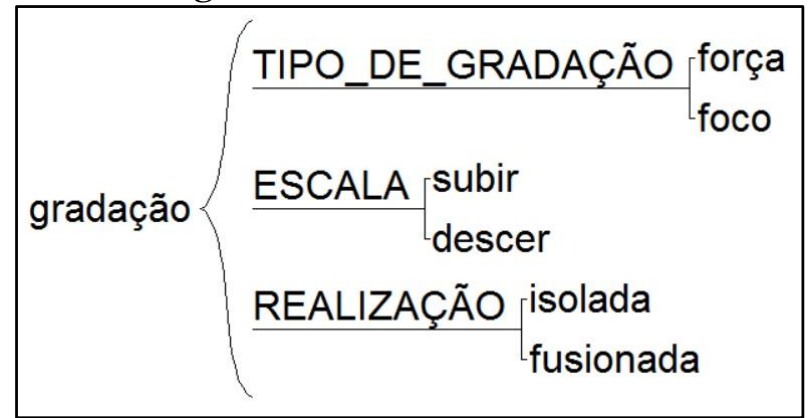

Fonte: Elaborado com base em Martin e White (2005). 
Da perspectiva da tradução interlinguística como reinstanciação do texto-fonte (SOUZA, 2010, 2013), os textos traduzidos são investigados em contraste com seus textos-fonte quanto ao acoplamento e à calibragem dos significados. O acoplamento se refere à integração de potenciais sistemas em cosseleção na instanciação dos significados, ou seja, quais dos sistemas potenciais são selecionados e combinados na construção dos significados. A calibragem, por sua vez, diz respeito ao grau de ativação do potencial de significação de cada sistema acoplado, ou seja, o nível de especificidade das escolhas em cada sistema acoplado.

Ressalta-se que foram apresentados aqui somente os conceitos basilares do modelo de língua da Linguística Sistêmico-Funcional, dos sistemas de VALORAÇÃo e da perspectiva de tradução como reinstanciação. Para aprofundamento, recomenda-se a leitura dos trabalhos referidos em cada tópico.

Em relação aos trabalhos dos Estudos da Tradução citados na Introdução deste, considera-se pertinente apresentar os resultados daquele que adotou a mesma perspectiva teórica-metodológica e o mesmo gênero, Cristófaro (2018). Além disso, são apresentados somente os resultados relativos às gradações, enfoque do presente estudo. Ao investigar dois textos-fonte e duas traduções de cada um deles, Cristófaro (2018) identificou que, de forma geral, variações as variações de acoplamento de calibragem da gradação nos textos traduzidos contribuíram para a intensificação dos valores de atitude, ou seja, houve aumento da gradação.

\section{METODOLOGIA}

Esta seção está dividida em duas subseções. Na primeira, são apresentadas narrativas selecionadas e seus critérios de seleção. Na segunda, são descritos os passos metodológicos adotados para o cumprimento dos objetivos deste estudo.

\subsection{NARRATIVAS}

Os textos selecionados para investigação neste estudo pertencem ao um dos subcorpora do Corpus de Traduções e Retraduções - RETRAD (MAGALHÃES, 2014). Trata-se de um subcorpus de textos ficcionais paralelos (originais em inglês e traduzidos em português). Foram selecionados dois textos traduzidos (TTs), O mundo se despedaça (2009) e A flecha de Deus (2011), por Vera Queiroz da Costa e Silva, e seus textos-fonte (TFs), Things Fall Apart (1994[1958]) e Arrow of God (1989[1964]), do escritor nigeriano Chinua Achebe. As capas das edições utilizadas são apresentadas na Figura 3. 


\section{das Letras}

Figura 3: Capas dos textos selecionados
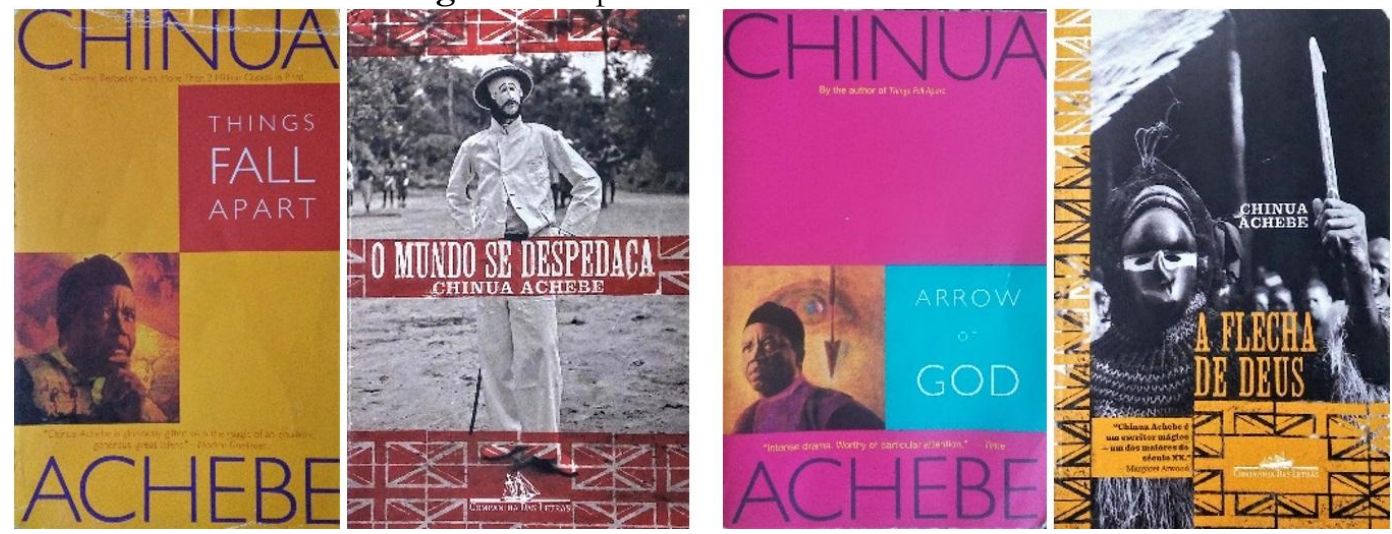

Fonte: Do autor (2018).

A seleção desses textos foi motivada pelos resultados de Dias e Magalhães (2017), que investigou um dos pares de textos (Arrow of God/ A flecha de Deus) com uma metodologia distinta e sugeriu aprofundamento da investigação. Além disso, considerou-se que o fato de as duas narrativas terem sido escritas pelo mesmo escritor e traduzidas pela mesma tradutora poderia ser produtivo para a identificação tanto de padrões linguísticos dos TFs quanto de padrões de variações nos TT's.

\subsection{ANÁLISE}

Inicialmente, cabe mencionar que os textos não foram analisados em sua totalidade. A análise abrangeu conjuntos de fases discursivas (MARTIN; ROSE, 2008) delimitados de diferentes estágios discursivos (MACKEN-HORARIK, 2003). Ressaltase também que, assim como em Dias (2018), Cristófaro (2018) e Dias e Magalhães (2017), adotou-se o uso de planilha eletrônica para agilizar o processo de classificação dos recursos de VALORAÇÃO.

A elaboração da planilha envolveu o estabelecimento de colunas duplicadas para as categorias da VALORAÇÃO, uma para o TF e a outra para o TT. As categorias foram: "comprometimento", "item valorativo", “avaliador", “afeto", “julgamento", "apreciação", "avaliado", "realização" e "gradação". Além disso, foram criadas listas de itens para as categorias com opções pré-definidas. No caso específico da gradação, as opções foram "força(+)", "força(-)", "foco $(+)$ " e "foco $(-)$ ", nas quais "(+)" significa grau subindo a escala e "(-)" significa grau descendo a escala. A Figura 4 apresenta a captura de tela da planilha utilizada. 
Figura 4: Captura de tela da planilha de análise da VALORAÇ̃̃O

\begin{tabular}{|c|c|c|c|c|c|c|c|c|}
\hline Texto-fonte & Item_valorativo & Avaliador & Afeto & Julgamento & Apreciação & Realizaçãc & - Avaliado & Gradação \\
\hline $\begin{array}{l}\text { Okonkwo was well } \\
\text { known throughout the } \\
\text { nine villages and even } \\
\text { beyond. }\end{array}$ & well known & narrador & 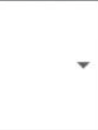 & (+)norm. & $\checkmark$ & inscrito & Okonkwo & força $(+)=$ \\
\hline \multirow{2}{*}{$\begin{array}{l}\text { His fame rested on } \\
\text { solid personal } \\
\text { achievements. }\end{array}$} & fame & narrador & $\checkmark$ & $(+)$ norm. $\quad$ & $\checkmark$ & inscrito & Okonkwo & $\checkmark$ \\
\hline & $\begin{array}{l}\text { solid personal } \\
\text { achievements }\end{array}$ & narrador & 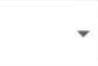 & (+)capac. - & $\checkmark$ & evocado - & Okonkwo & força(+) $=$ \\
\hline \multirow{3}{*}{$\begin{array}{l}\text { As a young man of } \\
\text { eighteen he had } \\
\text { brought honor to his } \\
\text { village by throwing } \\
\text { Amalinze the Cat. }\end{array}$} & honor & narrador & . & $(+)$ norm. & $\checkmark$ & inscrito & Okonkwo & . \\
\hline & throwing & narrador & $\checkmark$ & (+)capac. - & $\checkmark$ & evocado - & Okonkwo & $\checkmark$ \\
\hline & the Cat & narrador & $\checkmark$ & (+)capac. - & 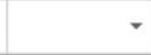 & evocado - & Amalinze & $\checkmark$ \\
\hline
\end{tabular}

Fonte: Do autor, 2018.

Antes de dar início às classificações, os TFs e TTs foram segmentados por proposição e inseridos nas planilhas em colunas pareadas, ou seja, uma proposição do TF ao lado da proposição equivalente do T'T. Esse procedimento permite o alinhamento dos textos e agiliza a identificação de variações.

Os procedimentos de análise da VALORAÇÃO foram baseados em Martin e White (2005), cujas orientações são apresentadas sinteticamente em Macken-Horarik e Isaac (2014) para a análise da atitude e da gradação. A análise do comprometimento foi feita também com base em White (2006).

Os passos metodológicos incluíram: 1) a classificação das proposições de acordo com os tipos de comprometimento, destacando em negrito os recursos de heteroglossia; 2) a identificação e classificação dos valores atitudinais explícitos, com sua carga valorativa, do alvo da avaliação (avaliado) e da fonte da avaliação (avaliador); 3) mesmo procedimento de 2) para os valores atitudinais implícitos; 4) a identificação e classificação dos recursos de gradação incidindo sobre os valores atitudinais; e 5) a identificação dos padrões configuracionais da VALORAÇãO nas diferentes fases, nos estágios discursivos e em diferentes textos.

Adicionalmente, segundo recomendação de Martin e White (2005), as classificações foram checadas em duplas de pesquisadores para validação da análise. $\mathrm{O}$ uso de planilhas eletrônicas também facilitou o compartilhamento das classificações entre os pesquisadores para essa checagem.

Por fim, as classificações dos TFs e T'Ts foram contrastadas para a identificação de variações, segundo os critérios de Souza (2010). As variações em que determinados recursos de gradação do TF não foram identificados no TT ou em que os recursos foram identificados somente no TT são consideradas variações de acoplamento. As variações de calibragem, por sua vez, são aquelas em que dois valores atitudinais equivalentes entre o TF e o T'T são graduados, mas em diferentes graus.

\section{RESULTADOS E DISCUSSÃO}

Esta seção está subdividida em duas partes. Na primeira, são apresentadas e discutidas as variações de gradação em que o grau de valores de atitude é ampliado, ou 
seja, valores em que houve aumento da gradação. As variações de gradação em que os valores atitudinais tiveram seu grau diminuído são apresentadas e discutidas na segunda subseção. Para identificação das narrativas, usa-se TFA para Things Fall Apart, MD para O mundo se despedaça, AG para Arrow of God e FD para $A$ flecha de Deus.

\subsection{VARIAÇÕES AUMENTANDO A GRADAÇÃO}

Inicialmente, é pertinente ressaltar que, embora as variações sejam apresentadas aqui em exemplos isolados, elas foram investigadas considerando as relações estabelecidas entre os valores atitudinais no desenrolar das fases discursivas analisadas. O Quadro 1 apresenta os exemplos de variação em que valores de atitude dos textosfonte foram intensificados.

Quadro 1: Exemplos de variações aumentando a gradação

\begin{tabular}{|c|c|c|c|}
\hline No. & TF & $\mathrm{TT}$ & Narrativa \\
\hline 1) & He breathed heavily, & Sua respiração era forte, pesada, & $\mathrm{TFA} / \mathrm{MD}$ \\
\hline 2) & $\begin{array}{l}\text { The eaves on this additional entrance were cut } \\
\text { back so that sitting on the floor Equlu could } \\
\text { watch that part of the sky where the moon had its } \\
\text { door. }\end{array}$ & $\begin{array}{l}\text { Os beirais dessa entrada adicional eram } \\
\text { tão estreitos que, sentado no chão, } \\
\text { Ezeulu podia vigiar aquela parte do céu } \\
\text { onde a lua tinha sua porta. }\end{array}$ & $\mathrm{AG} / \mathrm{FD}$ \\
\hline 3) & $\begin{array}{l}\text { If Ezenlu lived to be so old he too would } \\
\text { accept such a loss. }\end{array}$ & $\begin{array}{l}\text { Se Ezeulu chegasse a ser tão velho, } \\
\text { também ele aceitaria naturalmente tal } \\
\text { perda. }\end{array}$ & $\mathrm{AG} / \mathrm{FD}$ \\
\hline
\end{tabular}

Nota: Usa-se o negrito para destacar os recursos em que houve variação.

Fonte: Do autor, 2018.

Conforme consta no Quadro 1, foram identificadas três variações de aumento da gradação. Nos exemplos 1) e 2), o aumento da gradação incide sobre valores de apreciação (da respiração e dos beirais, respectivamente), enquanto no exemplo 3) o aumento incide sobre um valor de afeto, de inclinação da personagem.

A variação do exemplo 1) ocorreu em MD por meio da repetição de valores similares para avaliar um mesmo ente (a respiração). De uma perspectiva semântica da gradação, considera-se que um atributo (forte) ou o outro (pesada) já reinstancia o grau de intensificação de "heavily" no inglês. Assim, o uso de dois atributos com relativamente o mesmo grau de calibragem da gradação contribui para elevar ainda mais intensidade do valor atitudinal.

As variações dos exemplos 2) e 3) ocorreram na mesma narrativa e em um mesmo estágio discursivo. Nesses exemplos, valores atitudinais instanciados sem o acoplamento de gradação foram reinstanciados com gradação por meio de recursos isolados (tão e naturalmente). No caso do exemplo 2), houve ainda a explicitação do valor de apreciação, realizado por um atributo (estreitos), que no TF foi realizado de forma implícita (cut back), argumentativamente uma descrição de conteúdo ideacional. 


\section{das Letras}

No que concerne às implicações dessas variações no desenrolar da narrativa, pode-se argumentar que o acoplamento de gradação aos valores atitudinais contribui para a proeminência daqueles significados em sobreposição a outros. Essa maior proeminância tem o potencial de engajar o leitor prospectivo e alinhá-lo a determinado posicionamento de leitura.

\subsection{VARIAÇÕES DIMINUINDO A GRADAÇÃO}

Conforme mencionado, esta seção é dedicada à apresentação e discussão das variações em que os valores atitudinais tiveram seu grau diminuído. Os exemplos dessas variações estão dispostos no Quadro 2.

Quadro 2: Exemplos de variações diminuindo a gradação em ambas as narrativas traduzidas

\begin{tabular}{|c|c|c|c|}
\hline No. & TF & $\mathrm{T} T$ & Narrativa \\
\hline 4) & $\begin{array}{l}\text { Amalinze was a wily craftsman, but Okonkwo } \\
\text { was as slippery as a fish in water. }\end{array}$ & $\begin{array}{l}\text { Amalinze tinha uma destreza manhosa, } \\
\text { mas Okonkwo era tão escorregadio } \\
\text { quanto um peixe dentro d'água. }\end{array}$ & TFA/MD \\
\hline 5) & $\begin{array}{l}\text { They had broken into tumult instead of } \\
\text { action. }\end{array}$ & $\begin{array}{l}\text { O povo se entregara ao tumulto em } \\
\text { vez de agir. }\end{array}$ & TFA/MD \\
\hline 6) & $\begin{array}{l}\text { In this season of the year his task was not too } \\
\text { difficult; he did not have to peer and search the } \\
\text { sky as he might do when the rains came. }\end{array}$ & $\begin{array}{l}\text { Neste período do ano, sua tarefa não } \\
\text { era muito difícil, pois não se via } \\
\text { obrigado a }[\varnothing] \text { procurá-la no céu como } \\
\text { tinha de fazer na estação chuvosa. }\end{array}$ & $\mathrm{AG} / \mathrm{FD}$ \\
\hline 7) & $\begin{array}{l}\text { He peered more closely to make sure he was not } \\
\text { deceived by a feather of cloud. }\end{array}$ & $\begin{array}{l}\text { Apertou }[\varnothing] \text { os olhos para ter certeza de } \\
\text { que não estava sendo enganado por } \\
\text { uma pluma de nuvem. }\end{array}$ & $\mathrm{AG} / \mathrm{FD}$ \\
\hline 8) & $\begin{array}{l}\text { At any other time Ezeulu would have been more } \\
\text { than a match to bis grief. }\end{array}$ & $\begin{array}{l}\text { Em qualquer outra ocasião, Ezeulu teria } \\
\text { sido [Ø] capaz de suportar sua dor. }\end{array}$ & $\mathrm{AG} / \mathrm{FD}$ \\
\hline
\end{tabular}

Nota: Usa-se o negrito para destacar os recursos de gradação e o símbolo [Ø] para a não reinstanciação.

Fonte: Do autor, 2018.

De acordo com o disposto no Quadro 2, nota-se que o número de variações em que houve redução da gradação foi maior do que os de aumento da gradação (Quadro 1). Este resultado mostra uma tendência contrária à tendência identificada em Cristófaro (2018). Uma hipótese é que o aumento ou a redução da gradação seja uma característica particular de cada reinstanciação e não uma característica geral da tradução do inglês para o português. No entanto, são poucos os estudos com essa perspectiva de investigação da valoração para que essa hipótese se confirme ou não.

Um traço geral identificado nas variações do Quadro 2 é que as reduções de gradação incidiram sobre valores atitudinais que constroem o comportamento da 
personagem e não a estética ou as emoções afetivas. Outra constatação geral é que o número de variações identificadas em FD foi superior ao número de variações em MD.

As variações identificadas em $\mathrm{MD}$ são do mesmo tipo, redução de gradação fusionada. No TF, as escolhas lexicais "wily" e "broken into tumult" acoplam um grau de intensidade maior em relação às escolhas "clever" e "give into tumult", respectivamente. De acordo com pesquisas a corpora, dicionários e tesauros de língua portuguesa, as escolhas "manhosa" e "entregar-se ao tumulto" não acoplam o mesmo grau de intensidade que as escolhas do inglês. Essas duas variações ocorrem em valores acerca de personagens outras que não o protagonista, mas que, na análise das relações estabelecidas entre os valores atitudinais no desenrolar das fases discursivas, têm implicações para a construção retórica da narrativa. No caso do exemplo 4), a construção da personagem Amalinze como alguém altamente habilidoso serve o propósito de ampliar ainda mais a habilidade e a força do protagonista Okonkwo, que derrota Amalinze em uma luta. No exemplo 5), no desenrolar das fases do TF, a intensificação da falta de coragem daquelas pessoas serve para contrastar e ampliar a coragem do protagonista, avaliado como corajoso. No TT, a variação reduz a intensidade daquele valor atitudinal e, consequentemente, também reduz o contraste e a construção retórica da coragem do protagonista.

Em FD, as variações também seguiram um padrão, que foi o não acoplamento da gradação na reinstanciação daqueles valores. Recursos isolados que realizam gradação (peer, more, more than, remote) não foram reinstanciados em FD. A não reinstanciação da gradação desses valores resulta não somente na redução local da intensificação dos valores, mas também na redução da intensificação prosódica que se estabelece entre os valores intensificados no desenrolar das fases discursivas analisadas. Nos exemplos 6) e 7)), os valores intensificados no TF contribuem para a construção do elevado grau de precaução do protagonista Ezeulu no cumprimento de uma de suas funções mais importantes como líder daquela comunidade. Já no exemplo 8), a gradação acoplada ao valor de capacidade serve para contrastar o comportamento do protagonista antes e depois de determinado evento trágico na narrativa. Adicionalmente, no desenrolar das fases discursivas, serve também para intensificar a proporção da tragédia em si.

\section{CONSIDERAÇÕES FINAIS}

Este artigo apresentou um estudo de variações semânticas de VALORAÇÃO, mais especificamente de gradação, em conjuntos de fases discursivas de diferentes estágios de duas narrativas traduzidas do inglês para o português. Afiliado à perspectiva de Souza (2013) e Cristófaro (2018), as variações na tradução foram investigadas em relação ao acoplamento e calibragem dos significados reinstanciados.

Com base nos resultados obtidos, considera-se que a metodologia utilizada foi produtiva para o tipo de análise proposto. Considera-se também que os objetivos estabelecidos foram atingidos. Foram identificados os tipos de variações de gradação, os valores atitudinais relacionados às variações e as implicações dessas variações para o desenrolar das narrativas. Como tendência, os resultados mostraram que o maior 
número de variações foi de redução da gradação, tanto pelo não acoplamento quanto pela menor calibragem da gradação. Os principais valores atitudinais que tiveram sua gradação reduzida foram valores de julgamento, sobre o comportamento das personagens. Uma das principais implicações das variações identificadas foi a redução da proeminência de determinados valores, construída prosodicamente por meio das relações estabelecidas entre os valores graduados no desenrolar das fases discursivas.

É importante ressaltar este estudo explorou as variações de gradação somente em relação aos valores de atitude. Não foram exploradas as relações das variações na gradação desses valores com os tipos de comprometimento das proposições nas quais as variações ocorreram. Também não foram investigadas variações na gradação do comprometimento em si.

Para pesquisas futuras, sugere-se inicialmente a replicação deste estudo em outras narrativas para investigar se a hipótese de que o aumento ou a redução da gradação nos textos traduzidos é um traço particular de cada reinstanciação se confirma ou não. Sugere-se também ampliar a investigação das variações de gradação para os tipos de comprometimento.

Considera-se que este estudo contribui para o nicho de análise do produto dos Estudos da Tradução ao apresentar uma metodologia robusta de investigação de variações semânticas com base em critérios linguísticos. Além disso, os próprios resultados encontrados são também uma contribuição, pois ampliam os achados acerca desse tipo de variação e podem, juntamente com outros estudos, contribuir para a identificação de tendências mais generalizadas.

\section{REFERÊNCIAS}

ACHEBE, C. Arrow of God. 2. ed. New York: Anchor Books, 1989. . Things Fall Apart. New York: Anchor Books, 1994.

. O mundo se despedaça. 2. ed. Tradução de Vera Queiroz da Costa e Silva. São Paulo: Companhia das Letras, 2009. Título original: Things Fall Apart.

- A flecha de Deus. Tradução de Vera Queiroz da Costa e Silva. São Paulo: Companhia das Letras, 2011. Título original: Arrow of God.

BLAUTH, T. A paisagem indizivel em duas traduções brasileiras de Heart of Darkeness: uma análise de estilo com base em corpus. 2015. 138 f. Dissertação (Mestrado em Linguística Aplicada) - Faculdade de Letras, Universidade Federal de Minas Gerais, Belo Horizonte, 2015.

DIAS, C.; MAGALHÃES, C. Intervenção tradutória em textos literários: um estudo da apresentação da fala e da avaliação. Belas Infiéis, Brasília, v. 6, n. 1, p. 103-122, 2017.

HALLIDAY, M. An Introduction to Functional Grammar. London: Arnold, 1994.

HALLIDAY, M.; MATTHIESSEN, C. An introduction to functional grammar. 3. ed. 
London: Hodder Education, 2004.

MACKEN-HORARIK, M. Appraisal and the special instructiveness of narrative. Text, Adelaide, v. 2, n. 23, p. 285-312, 2003.

MACKEN-HORARIK, M.; ISAAC, A. Appraising Appraisal. In: THOMPSON, G; ALBA-JUEZ, L. (Ed.) Evaluation in context. Amsterdam/Philadelphia: John Benjamins Publishing Company, 2014. cap. 4, p. 67-92.

MAGALHÃES, C. Estra: um corpus para o estudo do estilo da tradução. Cadernos de Tradução, Florianópolis, v. 2, n. 34, p. 248-271, Jul./Dez. 2014.

MARTIN, J. English text. system and structure. Philadelphia/Amsterdam: John Benjamins Publishing Companies, 1992.

MARTIN, J.; ROSE, D. Working with discourse: meaning beyond the clause. London: Continuum, 2003. . Genre relations: mapping culture. London: Equinox, 2008.

MARTIN, J.; WHITE, P. The Language of Evaluation: Appraisal in English. New York: Palgrave MacMillan, 2005.

MUNDAY, J. Evaluation in translation: critical points of translator decision-making. London and New York: Routledge, 2012.

ROSA, A. Narrator Profile in translation: work-in-progress for a semi-automatic analysis of narratorial dialogistic and attitudinal positioning in translated fiction. Linguistica Antverpiensia, Antwerp, n. 7, p. 227-248, 2008.

ROTHERY, J.; STENGLIN, M. Entertaining and instructing: exploring experience through story. In: CHRISTIE, F; MARTIN, J. R. (Ed.). Genre and Institutions: social processes in the workplace and school. London and New York: Continuum, 1997. p. 231-263.

SOUZA, L. Interlingual re-instantiation: a model for a new and more comprehensive systemic functional perspective on translation. 2010. 339 f. Tese (Doutorado em Língua Inglesa) - Departamento de Língua e Literatura Estrangeiras, Universidade Federal de Santa Catarina, Florianópolis, 2010.

- Interlingual re-instantiation: a new systemic functional perspective on translation. Text\&Talk, Berlin, v. 33, n. 4/5, p. 575-594, 2013. 